Ann. Zootech., I978, 27 (2), I27-I37.

\title{
Influence respective de la fréquence quotidienne des évacuations mammaires et des stimulations du pis sur l'entretien de la sécrétion lactée chez la Brebis
}

\author{
J. IABUSSI İRE* ,J.-F. COMBAUD* et P. PÉTREQUIN** \\ *Laboratoire de Recherches sur la Traite, \\ Centre de Recherches de Rennes, I.N.R.A., \\ 35042 Rennes Cedex (France) \\ **Domaine expérimental de Brouessy, \\ Magny-Les-Hameaux, 78470 St-Rémy-les-Chevreuse (France
}

\section{Résumé}

Cinquante-sept brebis Préalpes du Sud, allaitant toutes des agneaux "étrangers ", sont réparties dans 3 lots présentant les caractéristiques suivantes:

Lot A : Système nerveux mammaire intact; 7 tétées/jour sur la moitié gauche du pis (Ag) et $2 /$ jour sur la moitié droite (Ad).

Lot B : Système nerveux mammaire intact; 2 tétées/jour sur les moitiés gauche (Bg) et droite (Bd).

Lot C : Mamelle totalement dénervée - 7 tétées /jour sur la moitié gauche $(\mathrm{Cg})$ et $z /$ jour sur la moitié droite Cd.

Ainsi, sur chaque demi-glande, le nombre "d'évacuations "mammaires correspond toujours au nombre de tétées (soit 2 pour $\mathrm{Ad}, \mathrm{Bd}, \mathrm{Bg}$, Cd et 7 pour $\mathrm{Ag}$ et $\mathrm{Cg}$ ) alors que le nombre de "stimulations " des cellules secrétrices dépend de celui des décharges hormonales galactopoiétiques même si celles-ci ont été induites par l'excitation du trayon contralatéral (soit o pour Cd et $\mathrm{Cg}$, 2 pour Bd et Bg et 7 pour $\mathrm{Ad}$ et $\mathrm{Ag}$ ).

Pour chacun des traitements, la production moyenne journalière calculée après $2 I$ jours d'expérience est rapportée au tableau 2 soit :

$$
\mathrm{Ad}=387 \mathrm{~g}, \mathrm{Ag}=597 \mathrm{~g}, \mathrm{Bd}=423 \mathrm{~g}, \mathrm{Bg}=4 \mathrm{I} 7 \mathrm{~g}, \mathrm{Cd}=24 \mathrm{I} \mathrm{g}, \mathrm{Cg}=425 \mathrm{~g} .
$$

Il ressort de l'analyse statistique accompagnant ces résultats que la galactopoï̀se :

- est fortement renforcée lorsqu'on accroît la fréquence quotidienne des "évacuations ";

- reste à un niveau modeste en l'absence de "stimulations " du pis (mamelle dénervée) mais n'est pas améliorée lorsque leur nombre quotidien est supérieur à 2 .

Ces observations confrontées aux documents bibliographiques rapportés dans la discussion, permettent de confirmer le rôle primordial de la vacuité mammaire dans l'entretien de la sécrétion du lait. 


\section{I. - Introduction}

iu cours d'une précédente étude (LABussitire, Combaud et Petrequin, I974), nous avons pu démontrer que la production laitière des brebis Préalpes du Sud augmente lorsqu'on accroît de I à 7 le nombre journalier de traites ou de tétées. Dans leurs grandes lignes, ces résultats confirment ceux obtenus dans l'espèce ovine avec des fréquences quotidiennes généralement plus faibles (le plus souvent de I à 3) par GaAi, (I957), McCance (I959), Bagdasarov (I960), Semjam (I96r), Grigorov et Shalichev (I962), Svec (1966), Morag (I966 et rg68), Damyanov et al. (I97I), Casu et Boyazoglu (I974) et Malossini (I975).

Ils concordent également avec cetix relevés chez les bovins (EILIOTT, I959) mais les causes de cette production plus importantes restent encore obscures puisque 2 explications principales et non nécessairement contradictoires, pet1vent être avancées.

Le métabolisme du tissu mammaire pourrait être activé :

I ${ }^{0}$ Par des évacuations répétitives du lait alvéolaire puisque l'accumulation prolongée du lait dans la glande et sa stagnation dans la lumière des acini freinerait la sécrétion des cellules situées à proximité (EDWARDS, I950, TVERSKOY, I955, Lakshmanan et al., I958, Denamur et Martinet, I959, Eliliot', I959, Clatesson et al., I959, Meites, Nicoli et TalWalker, I960, Linzell et Peaker, I971, Martinet, Morag et Denamur, i976).

$2^{0}$ Par les décharges plus fréquentes des principales hormones hypophysaires jouant un rôle dans l'entretien de la sécrétion. Il est en effet maintenant bien établi que des stimulations des terminaisons nerveuses de la mamelle induisent chez les ruminants domestiques non seulement la libération d'ocytocine (ELY et PETERSen, I94I) mais aussi celle de ACth, (Denamur, Stoliaroff et Desclin, I965) de GH (Har'T et Fl,Ux, I973; MarTal, I975) et de Prolactine (Bryant, Linzei, et GreeNwOOD, I970; JohKE, I969; FelL et al., I97I; MCNeILly, I972; Schaus, I972; HaRT, I975; KaNn, HaberT et Denamur, I973; ReinhardT et Schams, I974; Taranenko, Kasimov et Fedosimov, I973 et Kann et al., I977).

L'objet du travail que nous présentons ci-dessous est donc de mieux connaître l'influence respective des évacuations mammaires d'une part et des stimulations du pis associées aux décharges d'hormones galactopoïétiques d'autre part.

Nous avons cherché à dissocier ces 2 effets en utilisant des brebis à mamelle intacte ou dénervée dont chaque trayon est soumis séparément à diverses fréquences journalières de tétée.

\section{II. - Matériel et Méthodes}

\section{II.I. - Plan expérimental}

L'expérience porte sur 57 brebis Préalpes du Sud qui subissent entre le $25^{\mathrm{e}}$ et le $4^{6}{ }^{\mathrm{e}}$ jour de la période d'allaitement 1'un des traitements indiqués au tableau I. On remarquera que chaque jour pour chaque demi-mamelle : de tétées,

- le nombre d'évacuations mammaires correspond toujours au nombre 
TABLEAU I

Schéma expérimental réilisé pendant les $2 \mathrm{~T}$ jours de l'essai Caractéristiques des lots et des traitements

Experimental scheme used during the trial (2I days)

Characteristics of groups and treatments

\begin{tabular}{|c|c|c|c|c|c|}
\hline \multirow{2}{*}{$\begin{array}{l}\text { Nombre de brebis } \\
\text { (répartition en } \mathrm{I}^{\mathrm{re}}, 2^{\mathrm{e}} \text {, } \\
3^{\mathrm{e}} \text { et } 4^{\mathrm{e}} \text { lactation) } \\
\text { Number of ewes } \\
\text { (distribution inlactations } \\
\quad \mathrm{T}, 2,3 \text { and } 4 \text { ) }\end{array}$} & \multirow{2}{*}{$\begin{array}{l}\text { Lot } \\
\text { group }\end{array}$} & \multicolumn{3}{|c|}{$\begin{array}{l}\text { Nombre de } \\
\text { Number of }\end{array}$} & \multirow{2}{*}{$\begin{array}{c}\text { État de } \\
\text { la mamelle } \\
\text { Status of the } \\
\text { udder }\end{array}$} \\
\hline & & $\begin{array}{l}\text { tétées } \\
\text { sucklings }\end{array}$ & $\begin{array}{l}\text { stimulations } \\
\text { stimulations }\end{array}$ & $\begin{array}{l}\text { évacuations } \\
\text { evacuations }\end{array}$ & \\
\hline $2 \mathrm{I}(5, \mathrm{I}, \mathrm{II}, 4)$ & & $\begin{array}{l}7 \\
2\end{array}$ & $\begin{array}{l}7 \\
7\end{array}$ & $\begin{array}{l}7 \\
2\end{array}$ & $\begin{array}{l}\text { intacte } \\
\text { intact }\end{array}$ \\
\hline $20(5,2,9,4)$ & & $\begin{array}{l}2 \\
2\end{array}$ & $\begin{array}{l}2 \\
2\end{array}$ & $\begin{array}{l}2 \\
2\end{array}$ & $\begin{array}{l}\text { intacte } \\
\text { intact }\end{array}$ \\
\hline I6 $(3, I, 7,5)$. & . & $\begin{array}{l}7 \\
2\end{array}$ & $\begin{array}{l}0 \\
0\end{array}$ & $\begin{array}{l}7 \\
2\end{array}$ & $\begin{array}{l}\text { dénervée } \\
\text { dencruated }\end{array}$ \\
\hline
\end{tabular}

$\mathrm{g}:$ moitié gauche, left side; $\mathbf{d}:$ moitié droite, right side.

- le nombre de stimulations des cellules sécrétrices correspond à celui des décharges " probables " des hormones galactopoïétiques. Le chiffre retenu est de zéro lorsque la glande est dénervée et de 2 ou 7 lorsqu'elle est intacte. Dans ce dernier cas, il est toujours égal sur les deux moitiés de mamelle à la fréquence maximum de tétées, que celles-ci soient ipsi ou contre-latérales au trayon étudié.

\section{II.2. - Dénervation de la mamelle}

La technique utilisée est celle décrite par Denamur et Martinet (I959). Il s'agit d'une autogreffe sans section des vaisseaux inguinaux correspondant à un décollement mammaire suivi du rattachement de la glande sur la paroi abdominale. L'opération est effectuée en 2 temps (moitié après moitié) à 2 semaines d'intervalle 35 et $2 \mathrm{I}$ jours avant la mise bas.

\section{II.3. - Technique d'allaitement}

Les tétées sont assurées par des agneaux "étrangers" dont l'âge correspond approximativement au stade de lactation des brebis (soit entre 25 et 46 jours).

Les jeunes sont toujours séparés des mères sauf aux heures des tétées qui se situent à 7 heures et I 6 heures lorsque celles-ci sont biquotidiennes et à 4,7 , Io, 13, I6, I9 et 23 heures dans les autres cas. 
Nous avons choisi de ne pas utiliser l'agneau " maternel ", car celui-ci aurait difficilement supporté la pratique de 2 tétées par jour (lot Bd et Cd). Il aurait été handicapé dans sa croissance par rapport à celui tétant $7 \mathrm{f} /$ jour (lot $\mathrm{Ag}$ et $\mathrm{Cg}$ ) et à terme, la qualité des évacuations et des stimulations du trayon (par la langue du jeune) aurait pu en souffrir. En outre, les agneaux étrangers s'imposaient pour qu'à 7 heures et à 16 heures les 2 moitiés de mamelle soient vidées complètement et stimulées avec la même vigueur.

La production laitière de chaque quartier est appréciée en pesant les agneaux avant et après chaque tétée avec une précision voisine de 5 grammes.

Les contrôles sont réalisés les mardi, mercredi et jeudi de chacune des 3 semaines expérimentales.

\section{III. - Résultats}

Il ressort de l'examen du tableau 2 que :

Io Les moitiés gauche et droite de la mamelle ont des potentialités laitières équivalentes lorsqu'elles sont soumises au même traitement; en effet, avec 2 tétées de chaque côté la différence entre $\mathrm{Bd}$ ( $423 \mathrm{~g} / \mathrm{jour}$ ) et $\mathrm{Bg}$ ( $4 \mathrm{I} 7 \mathrm{~g} / \mathrm{jour}$ ) n'est pas significative. Ceci nous autorise donc à utiliser la technique de comparaison entre "quartiers " pour juger l'efficacité des différents types de sollicitations mammaires sur l'activité secrétoire.

$2^{\circ}$ Le passage de 2 à 7 tétées par jour avec des brebis à mamelles intactes se traduit par un accroissement de la production de $30,2 \mathrm{p}$. cent (4I) g respectivement pour les groupes $\mathrm{Ag}$ et $\mathrm{Bg}$ ) ce qui est très proche des valeurs obtenues précédemment (30, 9 p. cent, et 29,4 p. cent par LABussière, Combaud et PeTrequiN (I974) dans des conditions expérimentales différentes.

$3^{\circ}$ Lorsqu'on maintient constant le nombre de stimulations et que le nombre quotidien d'évacuations passe de 2 à 7 on observe une augmentation hautement significative du niveau de sécrétion :

- + 35, I p. cent sur les animaux à mamelle intacte avec 7 stimulations : comparaison $\mathrm{Ag}-\mathrm{Ad}$,

- + 43, I p. cent sur des animaux à mamelle dénervée avec o stimulation : comparaison $\mathrm{Cd} \leftrightarrow \mathrm{Cg}$.

De fréquentes vidanges de la glande jouent donc un rôle important pour une bonne galactopoïèse.

$4^{0}$ Lorsqu'on maintient constant le nombre de vidanges ( $2 /$ jour) on constate que la sécrétion n'est pas significativement affectée quand on passe de 2 à 7 stimulations (comparaison $\mathrm{Ad} \leftrightarrow \mathrm{Bd}$ ) alors qu'elle s'accroît de façon hautement significative entre o et 2 ou o et 7 stimulations quotidiennes (respectivement +42 , I p. cent et 37,7 p. cent pour les comparaisons $\mathrm{Cd} \leftrightarrow \mathrm{Bg}$ et $\mathrm{Cd} \leftrightarrow \mathrm{Ad}$ ).

On remarquera par ailleurs que l'augmentation de production entre o et 7 stimulations est sensiblement plus faible $(+28,8$ p. cent au lieu de $+37,7$ p. cent) lorsque l'analyse est effectuée avec un rythme constant de 7 évacuations par jour $(\mathrm{Ag} \leftrightarrow \mathrm{Cg})$.

L'ensemble de ces résultats, confirmés par l'analyse de variance et le test de Scheffe présentés au tableau 3, indiquent donc que la galactopoïèse : nale,

- reste à un niveau modeste en l'absence " probable " de décharge hormo- n'est pas améliorée lorsque leur nombre quotidien est supérieur à 2 . 
TABI,EAU 2

Effet du nombre de stimulations et d'évacuations mammaires sur la production journalière de chaque moitié de mamelle

Effect of the number of udder stimulations and evacuations on the daily mean production of each half udder

\begin{tabular}{|c|c|c|c|c|c|c|}
\hline & $\begin{array}{l}\text { Groupe } \\
\text { Ad }\end{array}$ & $\begin{array}{l}\text { Groupe } \\
\mathrm{Ag}\end{array}$ & $\begin{array}{l}\text { Groupe } \\
\text { Bd }\end{array}$ & $\begin{array}{l}\text { Groupe } \\
\mathrm{Bg}\end{array}$ & $\begin{array}{l}\text { Groupe } \\
\text { Cd }\end{array}$ & $\underset{\mathrm{Cg}}{\text { Groupe }}$ \\
\hline $\begin{array}{l}\text { Nombre de stimulations/jour } \\
\text { Number of stimulations/day }\end{array}$ & 7 & 7 & 2 & 2 & o & o \\
\hline $\begin{array}{l}\text { Nombre d'évacuations/jour } \\
\text { Number of evacuations/day }\end{array}$ & 2 & 7 & 2 & 2 & 2 & 7 \\
\hline $\begin{array}{l}\text { Nombre de données . . . . . } \\
\text { Number of data. . . . . . . }\end{array}$ & $2 \mathrm{I}$ & $2 \mathrm{I}$ & 20 & 20 & $x 6$ & I6 \\
\hline 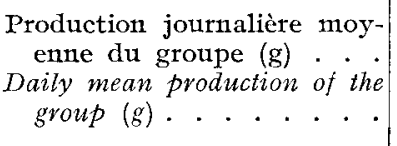 & 387 & 597 & $4^{23}$ & $4 \mathrm{I} 7$ & $24 \mathrm{I}$ & 425 \\
\hline Variance . . . . . . . . & II 599 & 23 I 86 & I3335 & I 2 or 2 & 2780 & Iо 266 \\
\hline $\begin{array}{l}\text { Comparaison } \\
\text { Comparison }\end{array}$ & Coms & $\begin{array}{l}\text { Comparais } \\
\text { s variance } \\
\text { arison of } v\end{array}$ & $\begin{array}{l}\text { n } \\
\text { riances }\end{array}$ & & $\begin{array}{l}\text { Comparais } \\
\text { s moyenne } \\
\text { barison of }\end{array}$ & $\begin{array}{l}1 \\
\text { ieans }\end{array}$ \\
\hline 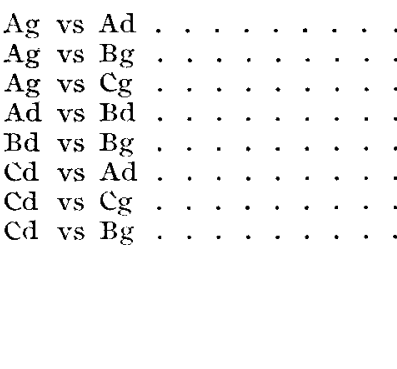 & 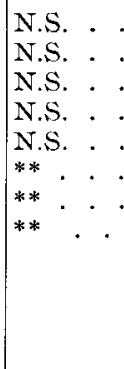 & 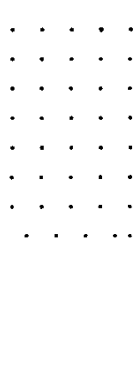 & $\begin{array}{l}F=2,00 \\
F=1,93 \\
F=2,25 \\
F=1,15 \\
F=1,11 \\
F=4,97 \\
F=3,69 \\
F=5,87\end{array}$ & 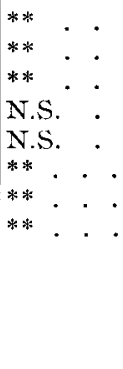 & 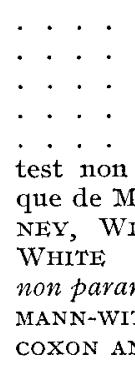 & $\begin{array}{l}t=5, \mathrm{x} 5 \\
t=4,36 \\
t=3,90 \\
t=1,03 \\
t=0,18 \\
\text { aarametri- } \\
\text { NN-WHIT- } \\
\text { CoXON et } \\
\text { étric test of } \\
\text { INEY WIL- } \\
\text { WHITE }\end{array}$ \\
\hline
\end{tabular}

NS : Non significatif. Non significant.

** : Significatif au seuil o,or. Significant at the o.or threshold.

(i) Iorsque les variances entre groupe sont différentes nous avons comparé les moyennes par le test non paramétrique de MaNN, WhITNEy, WILCOxON et WhITE qui ne fait appel à aucune hypothèse sur la nature des populations mises en jeu. (When variances between groups were different we compared the means by the non parametric test of MANN, WHINEY, WILCOXON and WHITE which does not appeal to any hypothesis about the nature of the populations involved.) 


\section{TABLEAU 3}

Effets de l'augmentation du nombre de stimulations mammairs sur le niveau de sécréiion dans le cas de a évacuations mammaires par jour

Effects of increasing the number of udder stimulations

on the secretion level in the case of 2 mammary evacuations per lay

\begin{tabular}{|c|c|c|c|c|c|c|}
\hline \multirow{4}{*}{$\begin{array}{c}\text { Analyse } \\
\text { de variance } \\
\text { des trois groupes } \\
\text { Ad, Bd, Cd } \\
\text { Analysis } \\
\text { of variance } \\
\text { of groups } \\
A d, B d, C d\end{array}$} & $\begin{array}{l}\text { Source } \\
\text { de variation } \\
\text { Source of variation }\end{array}$ & $\begin{array}{l}\text { Degrés } \\
\text { de liberté } \\
\text { Degrees } \\
\text { of freedom }\end{array}$ & $\begin{array}{l}\text { Somme } \\
\text { de carrés } \\
\text { Sum } \\
\text { of squares }\end{array}$ & \multicolumn{2}{|c|}{ Variance } & \multirow[t]{2}{*}{$\mathrm{F}$} \\
\hline & 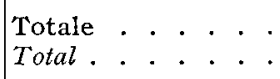 & 56 & 8477 Io & & & \\
\hline & $\begin{array}{l}\text { Entre traitement } \\
\text { Between treatment }\end{array}$ & 2 & 320712 & \multicolumn{2}{|c|}{ I60 355} & $\mathrm{I} 6,4^{* *}$ \\
\hline & $\begin{array}{l}\text { Erreut . . . . . . . } \\
\text { Error. . . . . . . }\end{array}$ & 54 & 526998 & \multicolumn{2}{|c|}{9759} & \\
\hline \multirow{3}{*}{$\begin{array}{c}\text { Test } \\
\text { de Scheffe } \\
\text { Scheffe's test }\end{array}$} & & $\begin{array}{c}\text { Groupe Ad } \\
7 \text { stimulations } \\
2 \text { évacuations } \\
3^{8} 7\end{array}$ & \multicolumn{2}{|c|}{$\begin{array}{c}\text { Groupe } B d \\
2 \text { stimulations } \\
2 \text { évacuations } \\
423\end{array}$} & & $\begin{array}{c}\text { Groupe } C d \\
\text { stimulation } \\
\text { évacuations } \\
24 \mathrm{I}\end{array}$ \\
\hline & $\begin{array}{l}\text { Groupe Ad } \\
\quad 3^{8} 7 \mathrm{~g}\end{array}$ & - & \multicolumn{2}{|c|}{$\begin{array}{l}36 \mathrm{~g} \\
\mathrm{NS}\end{array}$} & \multicolumn{2}{|r|}{$\underset{* *}{\mathrm{I} 46} \mathrm{~g}$} \\
\hline & $\begin{array}{l}\text { Groupe } B d \\
\quad 4^{2} 3 \mathrm{~g}\end{array}$ & - & \multicolumn{2}{|c|}{ - } & & I $82 \underset{* *}{g}$ \\
\hline
\end{tabular}

NS : Non significatif (Non significant.)

** : Significatif au seuil o,or. (Significant at the threshold o.or.)

\section{IV. - Discussion}

L'augmentation de production laitière constatée avec l'accroissement du nombre journalier de tétées s'explique à notre avis davantage par l'effet de vacuité résultant de l'élimination répétitive du lait alvéolaire que par le renforcement de la stimulation endocrinienne des cellules sécrétrices à la suite des sollicitations plus fréquentes de l'antehypophyse.

I. - L'évacuation fréquente du lait alvéolaire liée en partie à l'action galactokinétique de l'ocytocine permet l'entretien d'une abondante sécrétion

Il est en effet bien connu que la stagnation prolongée du lait dans la lumière des acini freine sensiblement la sécrétion (voir à ce sujet la revue d'ELLIOT'T, I959) 
mais, au vu de nos résultats et de ceux obtenus précédemment par d'autres chercheurs, on peut affirmer que ce phénomène persiste même si le tissu mammaire est abondamment imprégné du complexe hormonal galactopoïétique.

C'est ainsi que le réflexe neuroendocrinien de sécrétion décrit par SELYE (I934) chez la ratte n'empêche pas l'involution de la mamelle lorsque la ligature des trayons interdit aux jeunes d'extraire le lait. De même, WhEkLock, SMITH $\epsilon$ DoDD (I 667) observent, chez la même vache, le tarissement complet des quartiers non évacués alors que la sécrétion reste importante sur ceux traits normalement matin et soir.

Chez les bovins (Chafsson et al., I959) comme chez les ovins (MoRAG, I968), la production des demi-mamelles traites une fois par jour est sensiblemient plus faible que celles des moitiés qui le sont plus fréquemment.

De la même façon et bien qu'il induise probablement la décharge des hormones hypophysaires responsables de l'entretien de la sécrétion, le massage de la mamelle au milieu de chaque intervalle de traite ne provoque pas d'amélioration significative du niveau de production puisque ces stimulations supplémentaires ne sont pas suivies d'une évacuation du lait (GAUNyA et BUTLER, I960; MILLER et al., I963; LinNERUD et al., I966). C'est très certainement pour les mêmes raisons que l'ocytocine administrée entre les traites n'a que peu d'effet sur le niveau de sécrétion (CARoli et al., ig68; Martinet, Morag et Denamur, I976) alors qu'elle accroît sensiblement celui-ci lorsque l'injection est suivie de l'extraction immédiate du lait éjecté (1). C'est ainsi que DeAamur et Martinet (I96I) avaient déjà précédemment montré qu'après une traite de brebis considérée comme complète, 1'ocytocine à la dose de 5 UI, entraîne une augmentation de production $(+27 \mathrm{p}$. cent) uniquement sur le côté de mamelle où le lait résiduel est effectivement extrait.

Pour leur part LINZELL et PEAKER (I97I) observent un accroissement du même ordre lorsque des chèvres sont traites toutes les heures à l'aide de doses physiologiques de l'hormone posthypothysaire. Ces auteurs pensent toutefois qu'il faut moins imputer cet effet bénéfique à 1'action galactopoïétique de 1'ocytocine qu'à la grande fréquence des vidanges puisque, même en l'absence de traitement hormonal, ce rythme intense suffit à entretenir une abondante sécrétion sur des. glandes transplantées ne pouvant done plus transmettre d'influx nerveux.

On sait bien en effet, depuis les travaux de Denamur et MarTineT (I959) sur brebis et chèvres à mamelles totalement dénervées, que les stimulations à point de départ mammaire ne sont pas indispensables à 1'entretien de la sécrétion dans ces espèces. Mais, il est malgré tout probable que la suppression du réflexe d'éjection (qui se traduit par la disparition de la $2^{\mathrm{e}}$ émission sur les courbes de descentes du lait : LABUSSiÈre, MARTINET et Denamur, I969) est responsable de la baisse de production. Il nous faut en effet rappeler à cet égard que les brebis dites à une émission et ne fournissant naturellement à la traite que leur lait citernal, produisent moins (environ 20 p. cent) que les animaux à 2 émissions qui ne retiennent jamais leur lait alvéolaire (LABUssière, I966).

Des faits analogues ont été observés chez la vache puisqu'il suffit, pour obtenir un rendement normal, se remplacer les 2 traites journalières par la cathétérisation permanente des 4 trayons et l'évacuation biquotidienne des régions alvéolaires à l'aide de l'ocy tocine (MrEm KE et Brabant, I963). Au contraire, en se contentant de purger la citerne par une simple canulation TVERskoy (I955) aboutit chez la chèvre à une baisse de production de 20 à $40 \mathrm{p}$. cent et conclut comme le firent ultérieu-

1) Il semble bien malgré tout que l'ocytocine ait un effet galactopoićtique direct sur la mamelle puisqu'elle accélère la migration des protéines au sein même de la cellule sécrétrice (OLLIVIER-BousQueT, I976). 
rement Eilliot'T, I959; WhEELOCK, SMITH et DodD, I967; LiNZELI et PEAkER, I97I que la réduction de l'activité sécrétoire consécutive à l'accumulation du lait dans la mamelle n'est pas due à l'élévation de la pression intramammaire (ce que pensent EDWARDS, I950; LAKSHMANAN et al., I958 ou LINNERUD et al., Ig66) mais plutôt à la stagnation du lait dans la lumière des acini. Il est en effet possible, comme l'a montré LEVY (I964) que certains acides gras présents dans le lait résiduel puissent stopper la synthèse des matières grasses dans les cellules situées à proximité, et que d'une façon plus générale d'autres agents chimiques susceptibles d'être réabsorbés dans la voie sanguine (par exemple le calcium : GrEENBANM, GUMAA et MCLEAN, I97I) soient impliqués dans ces mécanismes d'inhibition.

\section{2. - Les hormones hypophysaires limiteraient la galactopoïèse lorsqu'elles sont libérées en quantité insuffisante, mais la multiplication des décharges aurait peu d'effet galactopoïétique.}

Le présent travail accordant une part plus importante à la fréquence journalière des évacuations qu'à celle des décharges endocriniennes pour expliquer l'effet bénéfique de l'augmentation du nombre de tétées est donc en parfait accord avec la plupart des observations expérimentales que nous venons de discuter. Nos résultats montrent toutefois qu'il ne faut pas éliminer pour autant la participation des facteurs hormonaux puisqu'en supprimant la totalité des stimulations mammaires (glandes dénervées) et une grande partie des autres stimulations sensorielles (les brebis sont tétées par des agneaux " étrangers ") le niveau de production est réduit probablement en relation avec celui des hormones circulantes galactopoiétiques.

Bien que nous n'ayons malheureusement pu doser ces dernières on peut néanmoins supposer que nos dénervations ont provoqué :

- une hypo-ocytocinémie se traduisant par une rétention lactée dont nous connaissons déjà les conséquences;

- une hypo-prolactinémie qui doit être tenue pour partiellement responsable de 1'activité plus réduite du tissu sécrétoire, comme KaNN et al., I977 1'ont par ailleurs montré chez la brebis en utilisant un inhibiteur spécifique de la décharge de prolactine la 2 -Bromo- $\alpha$-ergocryptine (CB I54);

- un abaissement des concentrations plasmatiques en hormone de croissance ce qui pourrait limiter chez la brebis l'obtention de hauts niveaux de production (Denamur et Martinet, I970).

Par contre, le passage de 2 à 7 stimulations journalières qui conduit certainement à des décharges plus fréquentes des hormones précitées ne se traduit pas par un effet significatif sur le volume de lait recueilli ce qui corroborerait les résultats de Scrams (I976) selon lesquels l'hyperprolactinémie ne provoque aucun changement de sécrétion chez les ruminants. Il ne faut pas oublier toutefois que la prolactine joue un rôle galactopoïtique secondaire dans ces espèces, mais il nous est par contre difficile d'expliquer pourquoi le rendement n'augmente pas entre 2 et 7 stimulations quotidiennes alors que l'injection de GH (BRUMBY et HANcock, I954) ou d'extraits hypophysaires totaux (FOLIEY, I954) sont à cet égard très efficaces.

Il nous faut pour conclure souligner que notre expérimentation reste criticable puisqu'il est délicat de comparer des animaux dénervés privés de stimulations mammaires a des animaux "témoins " tétés par des agneaux étrangers qui n’ont 
sans doute pas l'efficacité de stimulations des agneaux maternels. Ennfin, il importe de rester prudent dans l'interprétation des relations entre la teneur sanguine en hormone (que nous n'avons pu mesurer) et l'activité sécrétrice puisque celle-ci dépend très largement de la réceptivité des cellules cibles que nous ne connaissions pas.

Il nous semble donc opportun que des études complémentaires soient mises en place pour approfondir cette question.

Accepté pour publication en janvier 1978

\section{Summary}

\section{Infuence of the daily jrequency of udder evacuations and stimulations on the maintenance of milk secretion in the ewe.}

Fiftr-seven Préalpe du Sud ewes, all nursing "foreign " I ambs, were distributed into 3 groups subjected to the following treatments (Table $I$ ) :

Group A : Intact mammary nervous system, 7 sucklings per day on the left side of the udder (Ag) and 2 per day on the right side (Ad).

Group B : Intact mammary nervous system, 2 sucklings per day on the left (Bg) and right side (Bd).

Group C: 'Totally denervated udder, 7 sucklings per day on the left side (Cg) and 2 on the right side (Cd).

The number of mammary " evacuations " from each half-udder always corresponded to the number of sucklings (i.e. 2 for $\mathrm{Ad}, \mathrm{Bd}, \mathrm{Bg}, \mathrm{Cd}$ and 7 for $\mathrm{Ag}$ and $\mathrm{Cg}$ ) whereas the number of " stimulations " of the secretory cells depended on the number of galactopoietic hormonal releases even if the latter were induced by a stimulation of the contralateral teat (i.e. o for $\mathrm{Cd}$ and $\mathrm{Cg}$, 2 for $\mathrm{Bd}$ and $\mathrm{Bg}$ and 7 for $\mathrm{Ad}$ and $\mathrm{Ag}$ ).

Table 2 shows the daily mean production of milk calculated for each group after 2 I days of experiment:

$$
\mathrm{Ad}=387 \mathrm{~g}, \mathrm{Ag}=597 \mathrm{~g}, \mathrm{Bd}=423 \mathrm{~g}, \mathrm{Bg}=4 \mathrm{I} 7 \mathrm{~g}, \mathrm{Cd}=24 \mathrm{I} \mathrm{g}, \mathrm{Cg}=425 \mathrm{~g}
$$

A statistical analysis of the data (Tables 2 and 3 ) shows that the galactopoiesis

- was strongly enhanced when the daily frequency of " evacuations " increased,

- remained at a moderate level when no udder " stimulations " (denervated gland) were practised and was not improved when the daily number of stimulations exceeded 2 .

Comparison of these observations with the data from the literature reported in the discussion confirms the essential role of the manmary vacuity in the maintenance of milk secretion.

\section{Références bibliographiques}

BAGDASAROv G., I960. The milk production of fine wooled and Semi fin wooled ewes (en Russe). Oveevodstuo, 6, 22-24.

BRUMBy P. J., HANCOCK J., 1954. 'The galactopoietic role of growth hormone in Dairy Cattle. N.Z.J. Sci. Tech. Sec. A., 36 (5), 4I 7-436.

Bryant G. D., Linzel, J. I., GREENwOOD F. C., T970. Plasma prolactin in goats measured by radioimmunoassay. The effects of teat stimulation, mating behaviour, stress fasting and of oxytocin insulin and glucose injections. Hormones, 1, 26-35.

Carol, I. J., Jacobsex M. S., Kassouny M., Smitri N. E., Armstrong I.1., ig68. An inhibition effect of oxytocin on the milk ejection reflex. Endocrinology, 82, I79-I82.

Casu S., Boyazogit J. G., I 974. Effets de la suppression de la traite du soir chez la brebis Sarde. In Symposium sur la traite mécanique des petits muminants Millau. Ann. Zootech., No Hors Série, I $40-144$. 
Claksson O., Hansson A., Gustalisson N., Brannang E., 1959. Studies on monozygous cattle twins XVII. Once a day milking compared with twice a day milking. Acta. Agric. Scand., 9, $3^{8-58 .}$

Damyanov A., K'snedelcheva S., Minev I., Vasilev N., i97r. Machine milking of Maritsa Finerwool ewes of the Schr'klevo cooperative farm in Ruse district. Zhivotnov'dstvo, 25 (Io), 7-9 (in Dairy Sci. Abstr., I974, 36, 3788).

DENAmUR R., MARTinet J., I959. Le rôle du système nerveux de la glande mammaire dans l'entretien de la lactation. Arch. Sci. Physio., 13, z7r-352.

Denamur R., Martinet J., I96r. Action de l'ocytocine sur la sécrétion du lait de brebis. Ann. Endocvin., 22, 776-78I.

Denamur R., Martiner J., I970. Nature des hormones anté-hypophysaires stimulantes de la sécrétion lactée chez la brebis normale. Arch. Inter Pharmac. Ther., 186 (I), I85-187.

Deinamur R., Stoliaroff M., Descirin J., I965. Effets de la traite sur l'activité corticotrope hypophysaire des petits ruminants en lactation. C. R. Acad. Sc. Paris, 260, 3I75-3 I 78.

EDWARDS J., I950. Factors influencing the relationship between the secretion of milk and fat. J. Agr. Sci., 40, Ioo.

EII.IOT' G., 1959. The direct effect of milk accumulation in the udder of the dairy cow upon milk secretion rate. Dairy Sci. Abstr., 21 (ro), 435-439.

EIy F., PETERSEN W. E., I94I. Factors involved in the ejection of milk. J. Dairy Sci., 24, 2 I I223.

Feli, L. R., Beck C., Blockitey M. A., Brown J. M., Cat' K. J., Cummtng I. A., Goding J. R., I97 I. Prolactin in the dairy cow during suckling and machine milking. J. Reprod. Fertil, 24, I 44-1 45 .

ForLEY S. J., I954. Recherches récentes sur la physiologie et la biochimie de la sécrétion lactée in Actualités Biochimiques, No I8, Masson Ed. Paris.

GaAr, M., I957. La traite 3 fois par jour chez la brebis Tsigaï. Résultats obtenus dans une grande entreprise agricole. Allatenyesztes, 6 (I), 6I-64.

GAUNyA W. S., BuThER C. E., I960. Effect of extra udder stimulation on milk production. J. Dairy Sci., 43, I 890 .

Greenbanm A. L., GumaA K. A., Mc LEAN, I97I. The control of pathways of carbohydrate metabolism (in Symposium on Lactation University of Nottingham. Butterworth London Ed.).

Grigorov H., Shalichev Y., I962. The effect of the number of milking and of the different interval between them of the butter and protein content of the sheep milk obtained. Int. Dairy Congr. Section $A, 258-264$.

HART I. C., 1975. Concentrations of prolactin in serial blood samples from goats before during and after milking throughout lactation. $J$. Endocr., 64, 305-312.

HAR'r I. C., FLUX D. S., 1973. The release of growth hormone in response to milking in the goat during early and late lactation. J. Endocr., 57 (1), I $7^{-1} 7^{8}$.

Jонке $T$., I969. Prolactin release in response to milking stimulus in the cow and goat estimated by radioimmunoassay. Endoct. Jap., 16, I $79-185$.

KANN G., HABERT R., DENAMUR R., I973. Concentrations plasmatiques de la prolactine et de l'hormone thyréostimulante au cours de la traite des brebis : comparaison avec les effets du T.R.H. C. R. Acad., Sci., Paris, D 276, I 32 I-I 324.

Kann G., Habert R., Meusnier C., Ryniewicz H. S., i977. Prolactin release in response to nursing of milking stimulus in the ewe. Is it mediated by thyrotrophin releasing hormone. Ann. Biol. anim. Bioch. Biophys, 17 (3B), 44I-452.

Kann G., Carpentier M.-C., Fenre J., Martinet J., Maubon M., Meusnier C., Paly J., VerMEIRE N., I977. I actation and prolactin in sheep, role of prolactin in intitiation of milk secretion. In Int. Symposium on Prolactin. Nice (sous presse).

LABUSSIÈrE J., I966. Relations entre le niveau de production laitière des brebis et leur aptitude à la traite. XVII e Congr. Int. Laiterie, Munich, Section AI-435I.

I,Abussière J., Combaud J. F., Pétrequin P., I974. Influence de la fréquence des traites et des tétées sur la production laitière des brebis Préalpes du Sud. Ann. Zootech, 23, $445^{-457}$.

Labussière, J., Martinet J., Dexamur R., I969. The influence of the milk ejection reflex on the flow rate during the milking of ewes. $J$. Dairy Res., 36, I9I-20I.

Lakshmanan S., Shaw J. C., Mc Doweli, R. E., Ellmore M. F., Fohrman, i958. Short interval milking as a physiological technique. I. Effect of frequent milking with the aid of oxytocin on milk and milk fat production. J. Dairy $S c i ., 41$, I60I-1608.

LEVY R., I964. The effects of weaning and milk on mammary fatty acid synthesis. Biochim. Biophys. Acta., 84, 229-238. 
Linnerdd A. C., Caruolo E. V., Millek (i. F., Marx G. D., Donker J. D., Ig66. Lactation studies $-\mathrm{X}$. Total daily production as affected by number of times milked, number of times stimulated and method of stimulation. $J$. Dairy Sci., 49, I 529-I532.

IINZEI, J. L., PEAkER M., 197I. The effects of oxytocin and milk removal on milk secretion in the goat. $J$. Physiol., 216, 717-734.

Mc Cance I., 1959. The determination of milk yield in the merino ewe. Aust. J. Agric. Res., $10,839-853$.

MC NEILLY J., 1972. Observations on the pattern of prolactin release during suckling in the ewe. J. Reprod. Fert., 31, 487-488.

ILALossini F., I975. Influenza dell'eliminazione di una mungitura o di due consecutive per settimana sulla produzione del latte nelle pecore sopravissane. Ann. Ist. Sper. Zootec., 8 (I), I $-\mathrm{I} 2$.

MIARTAL J., I975. Effets de la traite sur le taux plasmatique de 1'hormone somatotrope chez la brebis et chez la chèvre. C. R. Acad. Sci. Paris, 280, Série D, 197-200.

MLartinet J., MIorag M., Denamek R., 1976. The role of exogenous oxytocin in lactating ewe. Ann. Biol, anim. Bioch. Biophys., 16, 833-849.

Meites J., Nicol, C. S., TALWALKER P. K., I960. Local action of oxytocin on mammaty glands of postpartum rats after litter removal. Proc. Soc. Exp. Biol. N. Y., 103 (r), I I8-I22.

Mielke H., BRABANT W., I963. Lactogenese and galactopoese beim Rind ohne Sang, Melkoder andere exogene, zur Milkchejecktion führende Enterreize. Avch. Exp. Vet. Med., 16 (4), 909-919.

Mimler G. E., Iineferud A. C., Miarx G. D., Cartolo J. D., Donker J. D., Gerrits R. J., I963. Amount of milk obtained per day as affected by two equal milking intervals number of times stimulated and method of stimulation. J. Dairy Sci., 46 (6), 625-626.

MORAG M., I966. A reduction of milk yield associated with certain half udder milking techniques. J. Dairy Res., 33, 223-224.

MIORAG M., I968. The effect of varying the daily milking frequency on the milk yield of the ewe and evidence on the nature of the inhibition of milk ejection by Half-udder milking. $A \mathrm{mn} . Z O o$ tech., 17, 35 r-369.

OLLIVIER-BousqLet M., I976. Effet de l'ocytocine in vitro sur le transit intracellulaire et la sécrétion des protéines du lait. C. R. Acad. Sc. Paris, D 282, I3-г6.

REINHARDT V., Schams D., I974. Analysis of teat stimulations as specific stimulus for prolactin in cattle. Neuroendocrinology, 14, 289-296.

Schams D., 1972. Prolactin levels in bovine blood influenced by milking manipulation, genital stimulation and oxytocin administration with specific consideration to the seasonal variations. Acta. Endocr., 71, 684-696.

Scriams D., I976. Hormonal control of lactation in Breast leeding and the mother. Ciba liound Symposium, 45, 27-48.

Selye H., I934. Cité par Denamur R. et Martinet J., I959.

Semjam S., I961. The problem of milking frequency of ewes. Pol'nohospodarstvo Bratislava, 8 (2), I31-138 (in Dairy Sci. Abstr., I961, 7, 314).

SVEC J., Ig66. A contribution to the number of milking of coarse wool sheep (en Tchèque). Vedecke Prace Vyokumneho ustown ovciarskeho. V. Trencin III, 23I-245.

Taranenko A. G., Kasimov Z. N., Fiddosimov V. A., I973. Milk ejection reflex and prolactin blood content in cows. Trudy l'elikolukokogo Sel'khozimstituta, 29, 59-60 (en Russe) (In Dairy Sci. Abstr.)

TVERskoY G. B., I955. The role of pressure fluctuations in the udder cavity system in the stimulation of milk-secretion. Trud. Inst. Fiziol. I P Pavlova, 4, 6874.

Wheliock J. V., SMith A., DOdd F. N., I967. The effect of a temporary suspension of milking in mid lactation on milk secretion after the resumption of milking and in the following lactation. J. Dairy Res., 34, I5I-I6I. 